\title{
En memoria del Dr. Héctor Orrego Matte
}

\author{
Juan Pablo Roblero Cum ${ }^{1}$
}

\author{
In memory of Dr. Héctor Orrego Matte
}

Como Sociedad Chilena de Gastroenterología y Asociación Chilena de Hepatología queremos honrar la memoria del Dr. Héctor Orrego Matte, quien fue presidente de esta Sociedad entre los años 1964 y 1966. Fue un brillante médico hepatólogo, académico e investigador, que lamentablemente por la contingencia nacional y problemas ajenos a la medicina y a la academia, tuvo que desarrollar la mayor parte de su carrera en el extranjero.

Héctor Orrego nació en Santiago de Chile el año 1923. Su padre fue Héctor Orrego Puelma, destacado médico tisiólogo, profesor de la Facultad de Medicina de la Universidad de Chile, fundador del sanatorio para tuberculosos de San José de Maipo y fundador del actual Instituto Nacional del Tórax. Su madre, Blanca Matte, fue una mujer sabia, muy inteligente e inquieta intelectualmente. Amaba la literatura y fue quien lo inició desde pequeño en la lectura de los clásicos y probablemente fue quien más influyó en su pasión por la literatura ${ }^{1}$.

El Dr. Orrego desde temprana edad sintió la necesidad de conocer y tratar de comprender en profundidad todo lo que lo rodeaba. Fue una persona de vastísima cultura, de gran educación, un gran conversador y comunicador. Mantuvo siempre una gran inquietud intelectual, filosófica y existencial. Quienes lo conocieron cuentan que sus argumentaciones siempre fueron un aporte a la reflexión. Sus alumnos y quienes tuvieron la oportunidad de escuchar sus conferencias se asombraban de su claridad y de lo interesante que resultaban sus exposiciones. Un notable ejemplo de esta capacidad expositiva resultó de un encuentro casual entre Pablo Neruda y Héctor Orrego en Isla Negra. Neruda quería escribir para sus "Odas Elementales" un poema sobre algún órgano del cuerpo y le pidió su consejo, al igual como lo había hecho con otros conocidos especialistas en otras áreas de la medicina de la época. El Dr. Orrego, le explicó con gran simpleza las complejas funciones que realiza el hígado, necesarias para el correcto funcionamiento del resto del organismo. Finalmente, a partir de lo aprendido en esta conversación, Neruda expresó genialmente en su "Oda al Hígado" la importancia del noble órgano ${ }^{2}$.
La inquietud intelectual y existencial lo acompañó durante toda su vida. Buscó respuestas a sus interrogantes sobre la vida, el hombre y el universo, a través de un análisis racional y meticuloso de lo explorado por la humanidad a través diversas disciplinas ${ }^{3}$. Quienes no lo conocimos lo podemos descubrir a través de los libros que escribió en sus últimas décadas.

También, por supuesto fue muy riguroso y metódico en su trabajo profesional. Esto queda de manifiesto en su estudio "Alcoholic Liver Disease: Information in Search of Knowledge?", que fue publicado en la revista Hepatology el año 1981. En él escribió un exhaustivo análisis de lo conocido sobre la fisiopatología de la enfermedad hepática producida por alcohol y plantea algunas ideas de cómo podría abordarse de forma más integral toda esta información para futuros estudios. Para esta publicación cita nada menos que 356 referencias $^{4}$.

El Dr. Orrego realizó sus estudios de Medicina en la Universidad Católica de Chile. Formó parte de una de las primeras promociones de la Escuela de Medicina de esta Universidad. Fue compañero y amigo de toda la vida de médicos notables, que fueron parte de la historia de la Escuela de Medicina de la Universidad Católica, tales como Juan de Dios Vial Correa, Joaquín Luco y Luis Vargas, entre muchos otros. Cuenta en sus libros que en la Escuela de Medicina, a diferencia de lo que fue su experiencia en el colegio, estudió con gusto por el deseo de aprender, transmitido por la pasión de sus docentes.

Se dedicó con gran entusiasmo los primeros años de médico a la investigación en fisiopatología y a formarse como gastroenterólogo. Realizó estudios de especialización en Filadelfia y Nueva York durante 2 años, y posteriormente un año en Inglaterra. Como médico, la investigación y la docencia fueron sus grandes pasiones. Fue profesor en la Universidad Católica desde 1954 a 1976 y simultáneamente fue Profesor Titular de la Escuela de Medicina de la Universidad de Chile hasta 1973. Fue fundador del Servicio de Gastroenterología del Hospital Clínico de la Universidad de Chile, donde además formó el Laboratorio de Investigación de Gastroenterología e inició la formación de Postgrado en Gastroenterología.

\author{
Departamento de \\ Medicina. \\ Sección de \\ Gastroenterología \\ Hospital Clínico de \\ la Universidad de \\ Chile. \\ Presidente de la \\ Asociación Chilena \\ de Hepatología. \\ Recibido: 19 de \\ octubre de 2020 \\ Aceptado: 5 de \\ noviembre de \\ 2020 \\ Correspondencia a: \\ Dr. Juan Pablo \\ Roblero \\ Cum
}




\section{Gastroenterología y algo más}

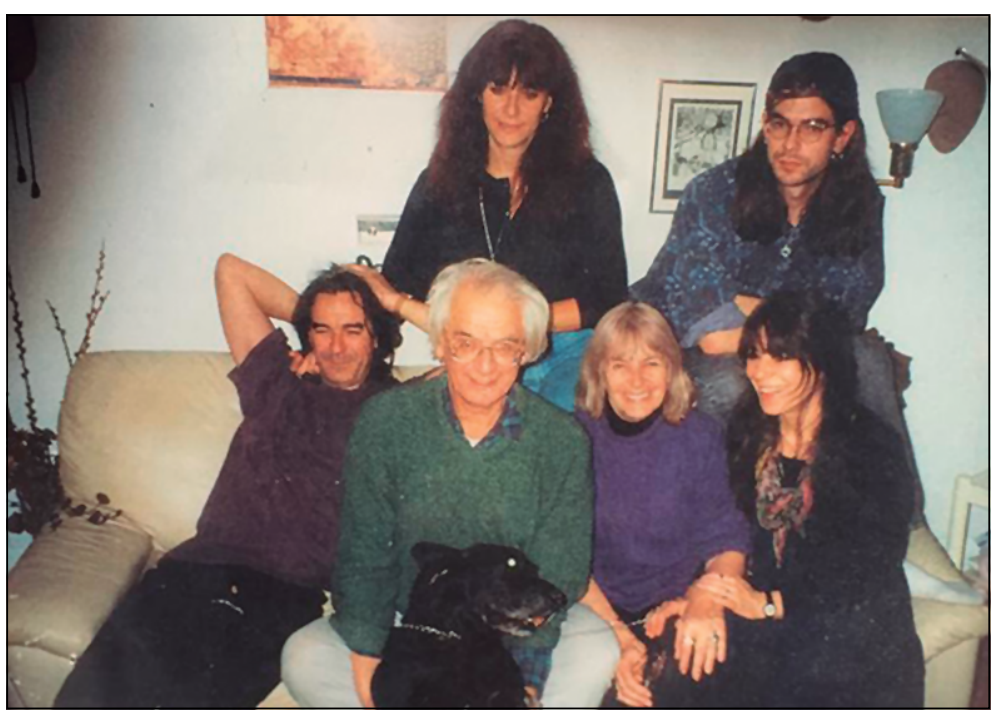

El Dr. Héctor Orrego junto a su Sra. Manena Silva y a sus hijos Felipe, Elena, Josefa y Pedro.

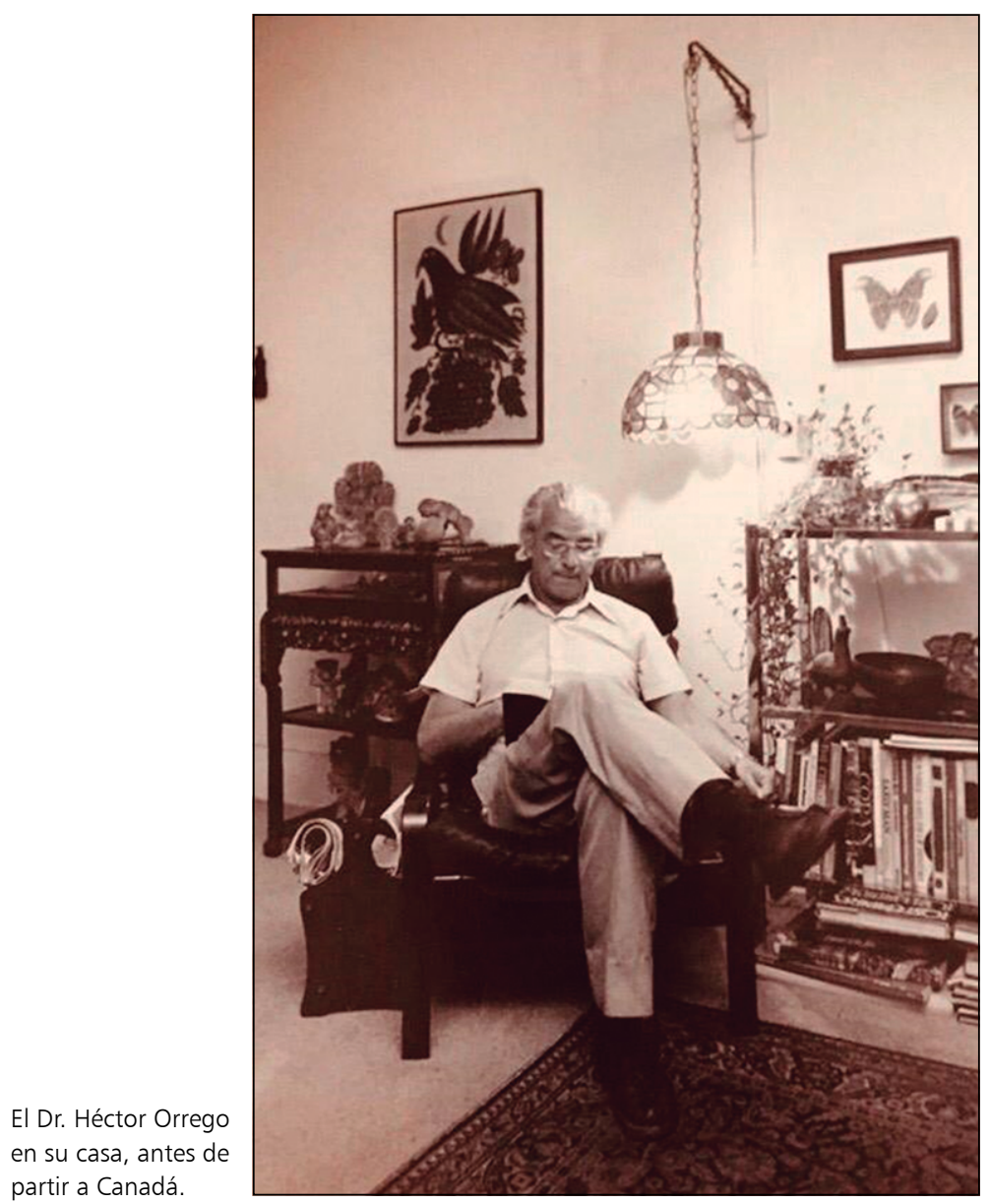

Fue vicedecano de la Facultad de Medicina de la Universidad de Chile, mientras era decano el Dr. Alfredo Jadresic. Junto a él y a otros muchos profesionales participó activamente en la Reforma Universitaria del año 1968.

Después del golpe militar en 1973 se vio obligado a buscar asilo en la embajada de Honduras y posteriormente partió al exilio junto a su señora e hijos. En Honduras tuvo un paso fugaz. En un comienzo pensó dedicarse a la fotografía, pero luego trabajó como técnico médico y posteriormente como profesor de fisiología. Gracias al prestigio que tenía por su labor científica y académica, fue invitado a trasladarse a Canadá. Ingresó como profesor asociado al Departamento de Farmacología y Medicina de la Universidad de Toronto, lugar donde pudo desarrollar una carrera brillante como docente e investigador. También, trabajó con mucho entusiasmo en el Instituto de Investigación en Alcoholismo (Alcoholism and Drug Addiction Research Foundation) que en esos años dirigía otro reconocido chileno. Durante los años 70 y 80 , en este Instituto realizó numerosos trabajos de investigación y publicaciones, de ciencias básicas y clínicas, de altísima calidad en relación a la enfermedad hepática por alcohol. Durante sus años de trabajo en Canadá, tuvo una activa presencia en congresos internacionales y logró un gran reconocimiento a nivel internacional. Cuenta con alrededor de 250 publicaciones científicas, muchas de las cuales se encuentran en las revistas más prestigiosas de medicina, gastroenterología y hepatología, tales como New England Journal of Medicine, Lancet, Hepatology, Journal of Hepatology ${ }^{4-7}$, por mencionar algunas. Entre los numerosos aportes a la hepatología, se encuentran los trabajos en que evaluó los efectos del estado hipermetabólico y del daño hipóxico celular en la patogenia de la enfermedad hepática por alcohol ${ }^{4-6}$.

Fue nombrado Profesor Emérito de Medicina y de

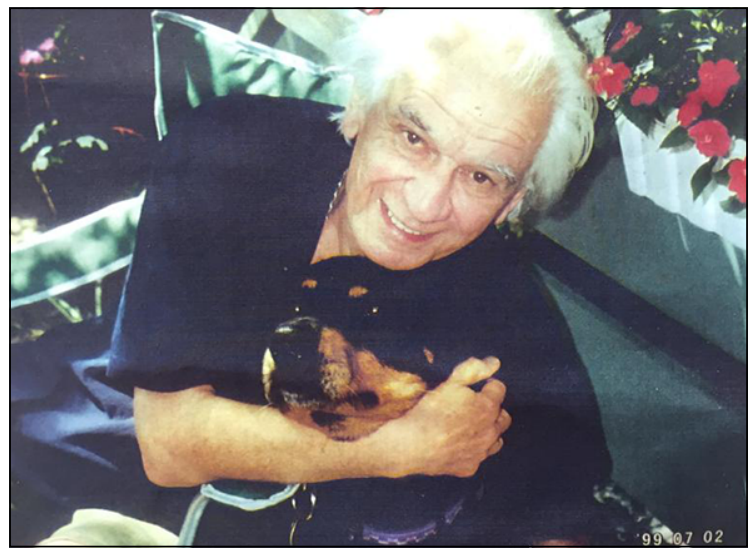

El Dr. Héctor Orrego junto a su querida perra Gita. 
Farmacología en la Universidad de Toronto, Canadá. También, fue nombrado Miembro del Royal College of Physicians and Surgeons of Canadá y en el año 1991 fue premiado con " The Canadian Gold Medal", otorgada por sus trabajos de investigación sobre los efectos del alcohol en el hígado.

Años después de jubilarse, retornó definitivamente a Chile el 2006 y no volvió a retomar actividades académicas, sin embargo se mantuvo muy activo del punto de vista intelectual, leyendo y escribiendo mucho, buscando como siempre respuestas a las grandes interrogantes del ser humano, con una mirada reflexiva, de asombro y con un ánimo de compartirlo y comunicarlo a sus lectores. Se podría decir que a través de esta forma siguió ejerciendo su pasión por la investigación y la docencia hasta los últimos años de su vida. En su libro titulado Bhudh-skö, palabra de origen indoeuropeo, que podría traducirse como búsqueda o "hacer algo para encontrar algo", podemos apreciar su profundo asombro por todo lo referente a la vida y al conocimiento alcanzado por la humanidad, además de su insaciable búsqueda de respuestas a las grandes interrogantes del hombre ${ }^{3}$.

Como Sociedad Chilena de Gastroenterología, lamentamos profundamente la partida del Dr. Héctor Orrego. Deseamos consuelo y paz a su señora, hijos, familiares y amigos.

Quiero expresar mis sinceros agradecimientos a la Sra. Manena Silva, su compañera de toda la vida y madre de sus cuatro hijos, quien me aportó valiosa información. También, doy mis agradecimientos a los amigos y colegas del Dr. Héctor Orrego que contribuyeron a la elaboración de este breve, pero sincero homenaje.

\section{Referencias}

1.- Orrego H. Mis años de aprendizaje. Santiago: LOM ediciones, 2010.

2.- Arrese M. The liver in poetry: Neruda's 'Ode to the liver'. Liver Int. 2008;28:901-5.

3.- Orrego H. Bhudh-skö. Tres divagaciones sobre la realidad. Santiago: LOM ediciones, 2005

4.- Orrego H, Israel Y, Blendis LM. Alcoholic liver disease: information in search of knowledge? Hepatology. 1981;1:267-83.

5.- Orrego H, Blake JE, Blendis LM, Compton KV, Israel Y. Long-term treatment of alcoholic liver disease with propylthiouracil. N Engl J Med. 1987;317(23):1421-7.

6.- Niemelä O, Risteli J, Blake JE, Risteli L, Compton KV, Orrego H. Markers of fibrogenesis and basement membrane formation in alcoholic liver disease. Relation to severity, presence of hepatitis, and alcohol intake. Gastroenterology. 1990;98 1612-9.

7.- Orrego H, Blake JE, Blendis LM, Kapur BM, Israel Y. Reliability of assessment of alcohol intake based on personal interviews in a liver clinic. Lancet. 1979;2: 1354-6. 